\title{
OS SENTIDOS DO RISCO NA GRAVIDEZ SEGUNDO A OBSTETRÍCIA: UM ESTUDO BIBLIOGRÁFICO
}

\author{
Romeu Gomes ${ }^{1}$ \\ Ludmila Fontenele Cavalcanti ${ }^{2}$ \\ Alice Salgueiro do Nascimento Marinho ${ }^{3}$ \\ Luiz Guilherme Pessôa da Silva ${ }^{4}$
}

Gomes R, Cavalcanti LF, Marinho ASN, Silva LGP. Os sentidos do risco na gravidez segundo a obstetrícia: um estudo bibliográfico. Rev Latinoam Enfermagem 2001 julho; 9(4):62-7.

O estudo objetiva analisar o conceito de gravidez de alto-risco, em artigos publicados nos anos 90, num periódico brasileiro representativo do campo da Ginecologia/ Obstetrícia. Em termos metodológicos, articulam-se as abordagens quantitativa e qualitativa, no estudo de 40 artigos. $A$ análise dos dados revela: uma diversidade dos sentidos atribuídos à gravidez de alto-risco e a redução do debate sobre o assunto. Dentre as conclusões, aponta-se a necessidade da perspectiva interdisciplinar na abordagem do tema, considerando a complexidade dos aspectos que envolvem a participação da mulher na atenção à gravidez.

PALAVRAS CHAVE: gravidez de alto risco, fatores de risco, risco

\section{THE MEANINGS OF RISK IN PREGNANCY ACCORDING TO OBSTETRICS: A BIBLIOGRAPHIC STUDY}

This study analyzes the meaning of high-risk pregnancy in articles published by a representative Brazilian journal in the field of Obstetrics in the 1990s. The methodological approach includes the quantitative and qualitative analysis of 40 articles, which reveals a diversity of meanings attributed to high-risk pregnancy and a reduced discussion on the theme. The conclusion shows the need to include an interdisciplinary approach to the theme by taking into account the complexity of the aspects involving women's participation in pregnancy care.

KEY WORDS: high-risk pregnancy, risk factors, risk

\section{LOS SENTIDOS DEL RIESGO EN EL EMBARAZO SEGÚN LA OBSTETRÍCIA: UN ESTUDIO BIBLIOGRÁFICO}

El estudio busca analizar el concepto de gravidez de alto riesgo, en artículos publicados en los años 90, en una revista brasileña representativa del campo de la Ginecología/Obstetricia. En términos metodológicos, se articulan los abordajes cuantitativo y cualitativo, en el estudio de 40 artículos. El análisis de los datos reveló: Diversidad de los sentidos atribuidos al embarazo de alto riesgo y un debate reducido a cerca del tema. Dentro de las conclusiones se enfatiza la necesidad de la perspectiva interdisciplinaria en el abordaje del tema, considerando la complejidad de los aspectos que envuelven la participación de la mujer en lo que se refiere al embarazo.

PALABRAS CLAVES: embarazo de alto riesgo, factores de riesgo, riesgo

\footnotetext{
${ }^{1}$ Doutor em Saúde Pública, Pesquisador Adjunto do IFF/FIOCRUZ; ${ }^{2}$ Mestre em Serviço Social; Docente da ESS/UFRJ; Coordenadora do Núcleo de Estudos e Ações em Saúde Reprodutiva; Doutoranda do IFF/FIOCRUZ; ${ }^{3}$ Mestre em Psicologia Social e Personalidade; Psicóloga do Departamento de Genética/IFF/ FIOCRUZ; Doutoranda do IFF/FIOCRUZ; ${ }^{4}$ Doutor em Medicina (Obstetrícia), Prof. Adjunto Fac. Med. da UFRJ ; Médico Obstetra do IFF/FIOCRUZ.Instituto Fernandes Figueira/FIOCRUZ - Av. Rui Barbosa, 716 - Rio de Janeiro - RJ - Brasil - CEP 22250-020
} 


\section{INTRODUÇÃO}

$E_{\text {timologicamente, a palavra risco é derivada do vocábulo }}$ "riscare", significando ousar. Assim, risco seria uma opção e não um destino(1).

Considera-se que as estimativas epidemiológicas de risco transcendem aspectos de sua construção metodológica, sendo, portanto, importante levar em conta as dimensões moral, política e cultural a elas relacionadas ${ }^{(2)}$.

Para o Ministério da Saúde ${ }^{(3)}$, a avaliação de risco não é tarefa fácil, uma vez que o conceito de risco associa-se a possibilidades e ao encadeamento entre um fator de risco e um dano nem sempre explicado.

Especificamente, o conceito de risco gravídico surge para identificar graus de vulnerabilidade nos períodos de gestação, parto, puerpério e vida da criança em seu primeiro ano ${ }^{(4)}$. Na assistência pré-natal, a gestação de alto-risco diz respeito às alterações relacionadas tanto à mãe como ao feto ${ }^{(5)}$.

Os estudos nacionais sobre o assunto, em geral, têm uma abordagem específica associada a um tipo de risco, não havendo uniformidade no tratamento do problema e acarretando dificuldade para se abranger o tema num só estudo, devido ao número de variáveis nele implíito ${ }^{(6)}$.

Devido à sua importância, a temática em questão vem sendo objeto de discussão no âmbito das políticas públicas em saúde e no campo das recomendações de procedimentos técnicos ${ }^{(7-10)}$. Para que se possa viabilizar tais políticas e procedimentos de uma forma mais efetiva, inicialmente faz-se necessário buscar os sentidos que envolvem a expressão gravidez de alto risco. Essa busca pode partir da Ginecologia/Obstetrícia, uma vez que se trata de um campo hegemônico quando se aborda tal assunto. Configurando-se os sentidos dessa área, os diferentes profissionais que integram a equipe de saúde na atenção às gestantes podem melhor compreender as ações médicas voltadas para essa problemática e, a partir disso, ampliar a discussão.

Com base nessas considerações iniciais, o presente estudo teve como objetivo analisar o conceito de gravidez de alto-risco, em artigos publicados no período 1990-1998, num periódico brasileiro representativo da área da Ginecologia/Obstetrícia. Nesse sentido, buscou-se identificar, numa parte da produção bibliográfica, os sentidos atribuídos a essa temática para que se possa ampliar 0 debate no campo da saúde materno-infantil, a partir de uma perspectiva multiprofissional.

\section{METODOLOGIA}

Foi selecionado um periódico de Ginecologia/Obstetrícia de abrangência nacional. Nesse periódico, foram analisados todos os artigos publicados no período de 1990 a 1998, que possuíam em seu título ou em suas palavras-chave as expressões gravidez ou gestação de alto-risco. Dentre os 723 artigos publicados no período, 40 se situavam nesse critério de inclusão. A análise do material fundamentou-se na articulação das abordagens quantitativa $e$ qualitativa, uma vez que as duas perspectivas se complementam na aproximação de uma realidade ${ }^{(11)}$.

Do ponto de vista da abordagem quantitativa, para cada artigo estudado, foram investigadas as seguintes variáveis: ano de publicação, palavras-chave, tipo de risco (fetal, materno e maternofetal), fatores de risco gestacional, metodologia utilizada. Essas variáveis, após codificadas, foram trabalhadas no Microsoft Access 97 e no Microsoft Excel 97.

Na classificação dos artigos por tipo de metodologia, optouse pela seguinte classificação: (a) Ensaio (estudo que se baseia unicamente na experiência do autor); (b) Estudo de caso clínico (estudo de casuística); (c) Estudo descritivo (estudo que não envolve metodologia epidemiológica, embora trabalhe os dados quantitativamente); (d) Estudo epidemiológico (estudo de distribuição de determinado fenômeno de saúde/doença em determinado local, espaço e grupo populacional); (e) Pesquisa qualitativa (estudo de crenças, valores, percepções, representações e sentidos atribuídos); (f) Revisão bibliográfica (estudo baseado em consulta bibliográfica); (g) Estudo quanti-qualitativo (estudo que articula as duas abordagens).

A análise dos artigos, de caráter qualitativo, baseou-se na Técnica de Análise de Conteúdo, modalidade Temática. A técnica de análise temática consiste em descobrir os 'núcleos de sentido' que compõem a comunicação e cuja presença, ou freqüência de aparição, podem significar alguma coisa para o objetivo analítico escolhido ${ }^{(12)}$. Com esta técnica, pode-se caminhar, também, na direção da descoberta do que está por trás dos conteúdos manifestos, indo além das aparências do que está sendo analisado ${ }^{(13)}$.

A partir desses princípios, basicamente, foram percorridos os seguintes passos de análise: (a) identificação das idéias centrais (núcleos de sentido) dos conceitos de gestação de risco de cada artigo; (b) comparação entre os diferentes núcleos de sentido presentes nos artigos estudados; (c) descoberta de eixos (temáticas) em torno dos quais giravam a construção dos conceitos e (d) discussão das categorias temáticas encontradas.

Por último, procurou-se articular os resultados de análise do banco de dados e os resultados da análise qualitativa.

\section{APRESENTAÇÃO E DISCUSSÃO DOS RESULTADOS}

O cenário da produção dos artigos

No período, foram consultados 89 fascículos, contendo 723 artigos. Desses artigos, 40 se enquadravam no objeto do presente 
estudo, correspondendo a 5,5\% do total dos artigos. A pouca produção pode ser relativizada se for levado em consideração o conteúdo dos artigos, em geral, e não apenas o título e as palavras-chave como critério de inclusão para fins de estudo. Assim, pode ser que um determinado artigo não tenha, em seu título ou em suas palavraschave, a indicação do termo "gravidez de alto-risco", mas o seu conteúdo se relacione a essa expressão.

Dos 40 artigos estudados, verificou-se que 17 (42,5\%) abordavam o risco fetal ${ }^{(15-31)}, 8(20 \%)$ tratavam de risco materno ${ }^{(32-}$ ${ }^{39)}$ e $15(37,5 \%)$ se relacionavam a risco materno-fetal ${ }^{(40-54)}$. Estes dados podem evidenciar a concomitância da exposição materna e fetal da gravidez de alto-risco (materno-fetal), justificando a abrangência do termo.

Ao se investigar os fatores de risco gestacional, constatouse que, do conjunto, 22 artigos (55\%) indicavam tais fatores, perfazendo um total de 68 fatores. Ao ser considerada a freqüência simples acima de 2, pode-se apontar o seguinte resultado: hipertensão arterial crônica (6), diabetes mellitus (5), cardiopatia (5), obesidade (4), crescimento intra-interino retardado (4). Nesses resultados, predominaram as intercorrências clínicas.

$\mathrm{Na}$ categorização desses fatores, levando-se em conta a classificação do Ministério da Saúde (9), como pode ser visto na Figura 1, obteve-se também a seguinte distribuição por tipo de risco: [a] intercorrências clínicas ( $n=53$ ), [b] características biopsicosocioculturais $(n=19)$, [c] história reprodutiva anterior $(n=12)$, [d] doença obstétrica na gravidez atual $(n=9)$. Apenas 3 fatores identificados pelos autores não foram categorizados de acordo com essa classificação (gemelaridade, hiperemesis, varizes de membros inferiores), o que foi denominado de resíduo. Essa distribuição admite respostas múltiplas, fazendo com que um mesmo artigo possa ser enquadrado em mais de uma categoria de risco. Observou-se no conjunto dos artigos a predominância de fatores de natureza clínica.

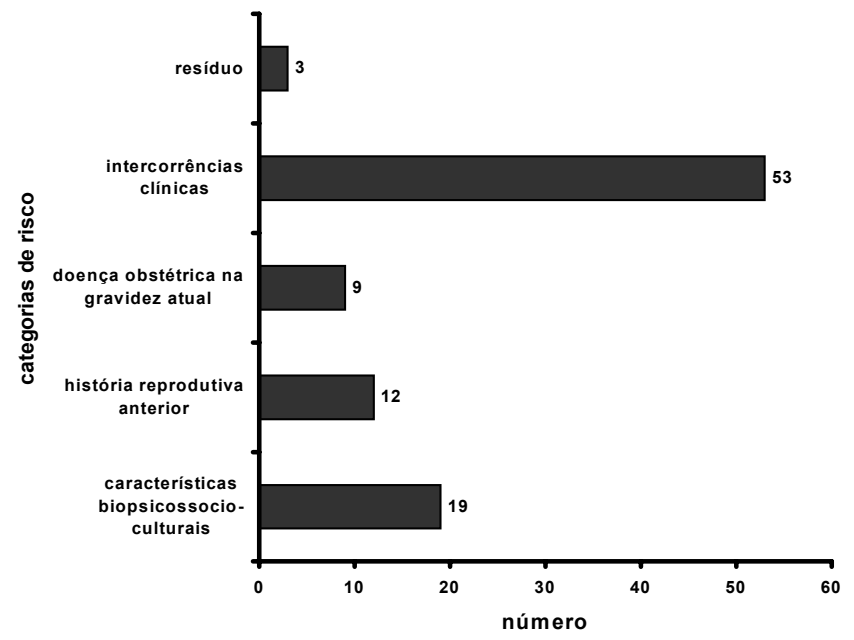

Figura 1 - Distribuição dos artigos segundo categoria de risco, 19901998
Em relação à metodologia adotada pelos autores dos artigos, conforme pode ser visto na Figura 2, houve uma predominância de estudos baseados em revisão bibliográfica (30\%), seguida do estudo de caso clínico $(27,5 \%)$ e do estudo descritivo $(22,5 \%)$.

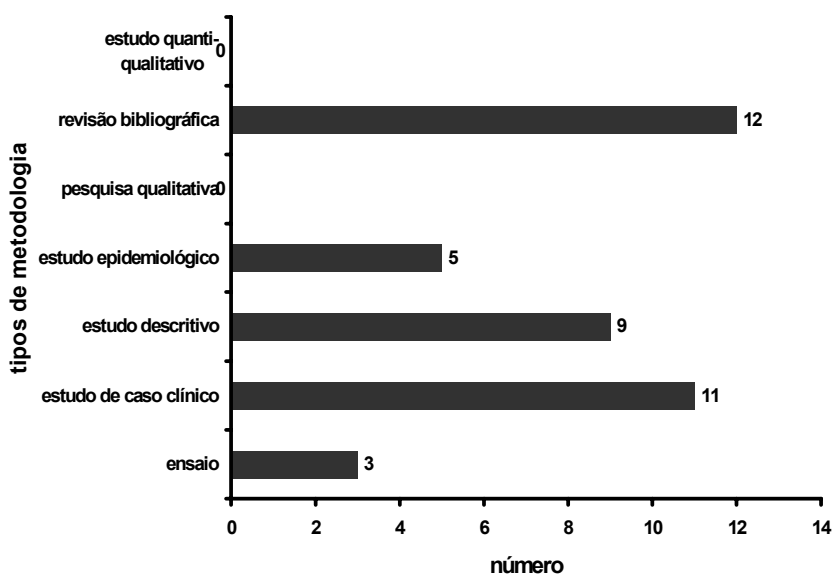

Figura 2 - Distribuição dos artigos segundo metodologia empregada, 1990-1998

A ausência de estudos qualitativos, observada na referida figura, pode corroborar a idéia reducionista no trato dos fatores de risco de caráter social que são de ordem mais complexa e menos precisa do que os fenômenos naturais ${ }^{(4)}$.

O significado de gravidez de alto-risco

Em geral, os artigos não trabalham o conceito de gravidez de alto-risco. Apresentam fatores que podem indicar um desfecho que compromete a gravidez, caracterizados como marcadores que visam à predição de morbi-mortalidade futura. Assim, durante a gestação, a mulher está sujeita a condições especiais consideradas inerentes ao estado gravídico, que acarretam mudanças nos processos metabólicos, que se medidas, podem determinar o estado fetal ${ }^{(14)}$. Seguindo esse raciocínio, toda gestação traz em si mesma risco para a mãe ou para o feto, no entanto, em pequeno número delas esse risco está muito aumentado ${ }^{(5)}$.

Observa-se que a idéia de probabilidade permite diferentes leituras e, nessa discussão, coexistem sentidos que vão desde 0 pólo objetivo, racional, preciso e mensurável, até o pólo intuitivo, subjetivo, vago e não mensurável ${ }^{(2)}$. Todavia, nesse vasto espectro, nota-se a presença da incerteza.

Ao se analisar os fatores de risco, pode-se, de uma forma indireta, chegar ao conceito de gravidez de alto-risco. Entretanto, pode-se perceber uma redução do conceito, uma vez que a idéia de fator de risco se associa mais a aspectos fisio-patológico, não abrangendo, necessariamente, outras dimensões do conceito de gravidez de alto risco. 
O Ministério da Saúde também vem concebendo os fatores geradores de risco numa maior amplitude do que a concebido pelos artigos estudados, classificando tais fatores em quatro grandes grupos: características individuais e condições sócio-demográficas desfavoráveis; história reprodutiva anterior à gestação atual; doenças obstétricas na gestação atual; e as intercorrências clínicas. A ausência de assistência pré-natal, por si mesma, é um fator de risco para a gestante e o recém-nascido ${ }^{(8)}$.

Numa primeira leitura dos artigos que abordam fatores de risco, podem ser identificados pelo menos cinco sentidos atribuídos à gravidez de alto-risco: complicação ${ }^{(33,40-44)}$, alteração ${ }^{(32,43,45-46)}$, anorma ${ }^{(15,40,47)}$, evolução desfavorável ${ }^{(47-48)}$, pejorativo ${ }^{(16)}$.

A diversidade dos sentidos atribuídos à gravidez de altorisco pode estar relacionada com a variedade de significados da expressão risco. Nesse sentido, a palavra risco assume diferentes sentidos e, em conseqüência disso, acarreta ambigüidades ${ }^{(2)}$.

Ao se considerar os sentidos de risco na gravidez, a discussão pode ser travada tendo como eixo questões associadas à patologia ${ }^{(55)}$. Num primeiro momento, os fenômenos patológicos, vistos como opostos ao normal, se constituem em variações que fogem de um padrão normativo no campo fisiológico. Segundo ele, a doença configura-se a partir da criação de padrão, de normas. Nesse sentido, a fisiologia foi concebida no meio normativo, sendo justificada pela necessidade de se conhecer para tratar.

A partir dessa perspectiva, os sentidos dos artigos complicação, alterações e evolução desfavorável podem ser traduzidos por situações que se afastam de um padrão previsível do processo gravídico, que fisiologicamente é considerado como uma etapa normal.

Em outro momento, ao analisar o conceito de patológico, toma-se como referência tanto o lugar da doença como o do doente, reivindicando o ponto de vista dinâmico da patologia, colocando a fisiologia em segundo plano e destacando a posição do doente ${ }^{(55)}$. Este sentido dinâmico não foi identificado no conjunto dos artigos estudados, ao contrário, a ênfase dada ao assunto baseava-se quase que exclusivamente no pólo fisiológico.

Em relação ao sentido pejorativo, a princípio esse termo pode causar um certo estranhamento, uma vez que no senso comum, é utilizado para algo que seja torpe e obsceno. Ao ser analisado o

\section{REFERÊNCIAS BIBLIOGRÁFICAS}

1. Bernstein PL. Desafio dos deuses - a fascinante história do risco. Rio de Janeiro (RJ): Campus; 1997.

2. Castiel LD. A medida do possível ... saúde, risco e tecnobiociências. Rio de Janeiro (RJ): Editora FIOCRUZ; 1999.

3. Ministério da Saúde (BR). Gestação de alto risco. Brasília (DF): Ministério da Saúde; 1995. verbo pejorar, no sentido de tornar pior ${ }^{(56)}$, esse termo pode fazer algum sentido, uma vez que foi empregado como evolução pejorativa para o concepto ${ }^{(16)}$. Nesse sentido, a expressão se aproxima à idéia da evolução desfavorável, situando-se também na mesma categoria de patológico.

A partir da análise dos artigos, fica patente que as idéias que giraram em torno da gravidez de alto-risco, podem ser reunidas num único núcleo de sentido: algo patológico em oposição a uma evolução considerada normal.

\section{CONSIDERAÇÕES FINAIS}

A partir da análise dos resultados, podem ser estabelecidas, pelo menos, duas conclusões: a imprecisão do que se entende por gravidez de alto-risco e a redução do debate. Em termos de redução, observa-se que, num primeiro momento, a gravidez de alto-riso fica reduzida a fatores, e, em segundo, o conteúdo dessa abordagem se limita, praticamente, aos aspectos fisio-patológicos. Tais reduções não facilitam a abordagem de questões relacionadas à mulher e à criança, enquanto sujeitos do ciclo gravídico.

Tanto a indefinição quanto a redução do assunto constatadas apontam para a necessidade de uma maior participação multiprofissional, para melhor conceituar, definir parâmetros, estabelecer procedimentos e prever medidas de prevenção acerca da gravidez de alto-risco.

Os conteúdos dos artigos reforçam a necessidade de se abordar a temática a partir de uma perspectiva interdisciplinar. A gravidez em si, já coloca a mulher numa situação de fragilidade, sujeita ao processo de medicalização, visto como uma ação centrada na condição orgânica em si ${ }^{(57)}$. No caso, ao ser acrescentada a idéia de risco, reforça-se a medicalização e a passividade de mulher frente a algo que possivelmente pode-Ihe ser adverso, mas que necessariamente não será. Diante dessa complexidade, a discussão não pode se restringir à clínica gineco-obstétrica, mas sim ampliar o debate para além do campo fisiológico, tentando compreender como a mulher vivencia esse processo e o que a leva a ter um determinado tipo de vivência.

4. Cesar CLG. O "enforque de risco" em saúde pública. In: Barreto ML, Almeida Filho N, Veras, RP, Barata, RB. Epidemiologia, serviços e tecnologias em saúde. Rio de Janeiro (RJ): FIOCRUZ/ABRASCO; 1998. p. 79-92.

5. Resende, J. Obstetrícia. $7^{\text {a }}$ ed. Rio de Janeiro (RJ): Guanabara Koogan; 1998.

6. Benathar LR. Contribuição ao estudo da relação entre algumas variáveis com a idade da gestação na matrícula em um serviço prénatal. [tese]. São Paulo (SP): Faculdade de Saúde Pública/USP; 1978. 
7. Ministério da Saúde (BR). Assistência pré-natal : normas e manuais técnicos. Brasília (DF): Ministério da Saúde; 1998.

8. Ministério da Saúde (BR). Gestação de alto risco. Brasília (DF): Ministério da Saúde; 2000.

9. CREMERJ (BR). Anteprojeto de atenção materno-infantil no Estado do Rio de Janeiro. Rio de Janeiro (RJ): CREMERJ; 1998.

10. OPAS/OMS. Prevenção e controle de enfermidades genéticas e os defeitos congênitos. Relatório de um grupo de consulta. Publicação científica n. 460. Washington (EUA): OPAS/OMS; 1984.

11. Minayo MCS, Sanches O. Quantitativo-qualitativo: oposição ou complementaridade? Cadernos de Saúde Pública 1993; 9(3):23962.

12. Bardin L. Análise de Conteúdo. Lisboa: Edições 70; 1979.

13. Gomes R. A análise de dados em pesquisa qualitativa. In: Minayo MCS. Pesquisa Social: teoria, método e criatividade. Petrópolis (RJ): Editora Vozes; 1994. p. 67-80.

14. Pinheiro N. Risco materno e risco fetal. Medicina Social 1999; 14(155):18-20.

15. Meirelles J Filho, Fonseca ALA, Amim J Júnior, Rezende J Filho, Chaves $\mathrm{H}$ Netto, Montenegro CAB. Reavaliação da doppler da artéria umbilical, na gestação de alto-risco, à luz da gasometria do sangue fetal obtido por cordocentese. Jornal Brasileiro de Ginecologia 1993; 103(3): 67-73.

16. Meirelles J Filho, Amim J Júnior, Fonseca, ALA, Resende J Filho, Chaves H Netto, Montenegro, CAB. Reavaliação do perfil biofísico fetal, na gestação de alto-risco, à luz da gasometria do sangue fetal obtido por cordocentese. Jornal Brasileiro de Ginecologia 1992a; 102(10):393-8

17. Amim J Júnior, Fonseca ALA, Chaves H Netto, Ximenes R. Rotinas atualizadas em medicina fetal - 4 - doppler-colorido. Jornal Brasileiro de Ginecologia 1995; 105(4):113-20.

18. Amim J Júnior, Lima MLA, Fonseca ALA, Bornia RBG, Chaves H Netto, Montenegro CAB. Dopplerfluxometria da artéria umbilical. Importância no diagnóstico do crescimento intra-uterino retardado na gestação de alto-risco. Jornal Brasileiro de Ginecologia 1991; 101(1-2):9-19.

19. Amim J Júnior, Fonseca ALA, Chaves H Netto, Junqueira JC. Rotinas atualizadas em medicina fetal - 6 - doença hemolítica perinatal. Jornal Brasileiro de Ginecologia 1995; 105(5):177-82.

20. Bornia RBG, Amim J Júnior, Lima MLA, Fonseca ALA, Chaves H Netto, Montenegro CAB. Dopplerfluxometria da artéria umbilical na avaliação da vitabilidade fetal na gestação de alto-risco. Jornal Brasileiro de Ginecologia 1990; 100(8):225-36.

21. Britto-Pereira PO, Chaves H Netto, Montenegro CAB. Utilização do sonar-doppler no diagnóstico da aceleração da frequência cardíaca fetal. Jornal Brasileiro de Ginecologia 1992; 102(10):405-13.
22. Martins-Costa SH, Ramos JGL, Lisboa PV, Pederiva MA, Toniazzo MR, Vettorazzi J, et al. Uso de fármacos na gestação. Jornal Brasileiro de Ginecologia 1993; 103(10):371-8.

23. Feraz EM, Gomide S, Prado LH, Alves EA, França PS. Cardiotocografia basal: estudo da validade e aplicação do teste em hospital de nível secundário. Jornal Brasileiro de Ginecologia 1990; 100(11-12):419-24.

24. Fonseca ALA, Amim J, Chaves H Netto, Ximenes R. Rotinas atualizadas em medicina fetal 2 - estudo genético pré-natal. Jornal Brasileiro de Ginecologia 1995; 105(3):61-5.

25. Fonseca ALA, Amim J Júnior, Chaves H Netto, Junqueira JC. Rotinas atualizadas em medicina fetal. 5 - doença hemolítica perinatal. Jornal Brasileiro de Ginecologia 1995; 105(5):171-5.

26. Meirelles J Filho, Alvim J Júnior, Fonseca ALA, Resende J Filho, Chaves H Netto, Montenegro CAB. Reavaliação da cardiotocografia basal, na gestação de alto-risco, à luz da gasometria do sangue fetal obtido por cordocentese. Jornal Brasileiro de Ginecologia 1992b; 102(11-12):429-35.

27. Meirelles J Filho, Amim J Júnior, Fonseca ALA, Chaves H Netto, Montenegro CAB. Mortalidade perinatal na Maternidade-Escola da Universidade Federal do Rio de Janeiro no período de 1985 a 1989. Jornal Brasileiro de Ginecologia 1990; 100(8):243-7.

28. Meyer L, Souza JHK, Falcão JOA Júnior, Silva HMS. Valor da cardiotocografia nas gestações de alto risco. Jornal Brasileiro de Ginecologia 1996; 106(10):383-8.

29. Peixoto MAP, Chaves H Netto, Montenegro, CAB. Dopplerfluxometria: bases físicas. Jornal Brasileiro de Ginecologia 1990; 100(8):197-201. -

30. Roucourt S, Vázquez ML, Oliveira AL, Bertini AM. A profilaxia e os fatores que influenciam no trabalho de parto prematuro. Jornal Brasileiro de Ginecologia 1994; 104(11-12):413-7.

31. Vitral ZNR, Pereira AK, Leite HV, Cabral ACV. Avaliação da concordância entre o perfil biofísico fetal e seus parâmetros ecográficos e o doppler umbilical na avaliação da vitalidade fetal, em gestações de alto risco. Jornal Brasileiro de Ginecologia 1994; 104(5):145-8.

32. Cunha AA, Geller M, Setti YB, Correa CS, Araújo MCD, Pessoa RLH. Gestação de alto risco - avaliação de sua incidência. Jornal Brasileiro de Ginecologia 1990; 100(3-4):83-4.

33. Diniz LEV, Zugaib M. Obesidade e gravidez. Jornal Brasileiro de Ginecologia 1991; 101(3):51-4.

34. Boller A, Cabral ACV. Dopplerfluxometria das artérias uterinas na avaliação do prognóstico materno. Jornal Brasileiro de Ginecologia 1995; 105(1-2):31-4.

35. Chaves H Netto, Amim J Júnior, Fonseca ALA, Silva LGP. Rotinas atualizadas em medicina fetal 7 - crescimento intra-uterino retardado. Jornal Brasileiro de Ginecologia 1995; 105(10):425-9. 
36. Duarte G, Mussi-Pinhata MM, Del Lama J, Takeda E, Pasti MJ, Costa JC. Valor do questionário específico na identificação de parturientes de risco para infecção pelo vírus da imunodeficiência humana (HIV). Jornal Brasileiro de Ginecologia 1991; 101(5):16974.

37. Mendonça D, Mendonça F, Grisi JC, Silva FSM, Bezerra J Júnior, Gonçalves SM. Obesidade e Gravidez. Jornal Brasileiro de Ginecologia 1997; 107(1-2):17-22.

38. Silva AR Filho. Cardiopatia e gravidez: resultado gestacional. Parte I- adaptações cardiocirculatórias à gravidez, parto e puerpério. Jornal Brasileiro de Ginecologia 1991; 101(3):55-8.

39. Silva AR Filho. Cardipatia e gravidez: resultado gestacional. Parte II - a gestante cardiopata. Jornal Brasileiro de Ginecologia 1991; 101(5):185-91.

40. Araújo DAC, Zimmermmann JB, Oliveira LCN, Andrade ATL. Gestação de alto-risco: prevalência de patologias e complicações materno-fetais. Jornal Brasileiro de Ginecologia 1996; 106(8): 31520.

41. Chaves H Netto, Pereira JP Júnior, Montenegro CAB. Conduta obstétrica na gestante diabética. Jornal Brasileiro de Ginecologia 1991; 101(11-12):493-8.

42. Diniz LEV, Cristofoli JCN, Addes A, Zugaib M. Nefropatia diabética e gravidez. Apresentação de um caso. Jornal Brasileiro de Ginecologia 1991; 101(7):275-8.

43. Oliveira JA, Levy RA, Avvad E, Porto LC. Histologia placentária nas gestações de alto risco: lúpus eritematoso sistêmico e préeclâmpsia. Jornal Brasileiro de Ginecologia 1996; 106(9):323-34.

44. Viggiano MGC, Castro AVB, Barros AP, Garcia CP, Maia HCA, Soares RA, et al. A grande multípara - estudo retrospectivo de junho/ 80 a agosto/86 com 882 casos (comparativo com 1.939 casos de junho/70 a agosto/76. Jornal Brasileiro de Ginecologia 1991; 101(3):83-91.
45. Barreto JLP, Galo ALR, Carvalho BCJ. HTLV-I e gestação considerações. Jornal Brasileiro de Ginecologia 1998; 108(9):311-3. 46. Magalhães CEF, Costa AG, Medina PAE, Levy RA, Barreto JLP. Esclerodermia Sistêmica e gravidez - relato de caso. Jornal Brasileiro de Ginecologia 1997; 107(11-12):429-32.

47. Costa AG, Magalhães CEF, Medina EAP, Barreto JLP, Heuseler RAM. Doença de Takayasu e Gestação - relato de caso. Jornal Brasileiro de Ginecologia 1997; 107(1-2):31-3.

48. Santos OJ, Branco ML Neto, Bezerra JA, Netto AL, Sousa LANG Júnior, Rodrígues $A$, et al. Pancreatite aguda necrohemorrágica durante a gravidez. Jornal Brasileiro de Ginecologia 1996; 106(12):41-5.

49. Caetano JPJ, Roquete ALB, Nascimento LG, Leite HV, Marinho RM, Lopes GP, Silva HMS. Gestação após os 40 anos. Jornal Brasileiro de Ginecologia 1991; 101(8):339-41.

50. Levy RA, Barreto JLP, Costa TP, Papi JAS. $O$ acompanhamento da mulher com lúpus sistêmico nos períodos pré-gestacional, prénatal e puerperal. Jornal Brasileiro de Ginecologia 1993; 103(1112):393-401.

51. Pantaleão S, Nunesmaia HGS, Paiva CSM, Medeiros JG Filho. Risco gestacional na anemia falciforme. Jornal Brasileiro de Ginecologia 1992; 102(10):415-7.

52. Ramos JGL, Martins-Costa S, Barros EJG, Nascimento LB, Mulle LD, Vileger R. Doença renal e gestação. Jornal Brasileiro de Ginecologia 1993; 103(10):355-8.

53. Sá RAM, Brochini M Júnior, Chaves $H$ Netto. Toxoplasmose congênita. Jornal Brasileiro de Ginecologia 1997; 107(8):281-6.

54. Silva AR Filho. Cardiopatia e gravidez: resultado gestacional. Parte III - O parto. Jornal Brasileiro de Ginecologia 1991; 101(6):205-12.

55. Canguilhem G. 0 normal e o patológico. $4^{\mathrm{a}}$ ed. Rio de Janeiro (RJ): Forense Universitária; 1978.

56. Ferreira $A B H$. Novo dicionário da língua portuguesa. $1^{\text {a }} \mathrm{ed}$. Rio de Janeiro (RJ): Nova Fronteira; 1975.

57. Vieira EM. A medicalização do corpo feminino. In: Giffin K, Costa SH. Questões da saúde reprodutiva. Rio de Janeiro (RJ): Editora Fiocruz; 1999. p. 67-78. 